\title{
DISTORTION AND CONVOLUTIONAL THEOREMS FOR OPERATORS OF GENERALIZED FRACTIONAL CALCULUS INVOLVING WRIGHT FUNCTION
}

\author{
M. K. AOUF and J. DZIOK \\ Received July 9, 2007 and, in revised form, March 1, 2008
}

\begin{abstract}
Using the Wright's generalized hypergeometric function, we investigate a class $W(q, s ; A, B, \lambda)$ of analytic functions with negative coefficients. We derive many results for the modified Hadamard product of functions belonging to the class $W(q, s ; A, B, \lambda)$. Moreover, we generalize some of the distortion theorems to the classical fractional integrals and derivatives and the Saigo (hypergeometric) operators of fractional calculus.
\end{abstract}

\section{INTRODUCTION}

Let $\mathcal{A}$ denote the class of functions $f(z)$ of the form:

$$
f(z)=z+\sum_{k=2}^{\infty} a_{k} z^{k},
$$

which are analytic in the unit disc $U=\{z: z \in C$ and $|z|<1\}$.

2000 Mathematics Subject Classification. Primary: 30C45, 26A33.

Key words and phrases. Wright's generalized hypergeometric function, modified Hadamard product, fractional calculus.

ISSN 1425-6908（C) Heldermann Verlag. 
If $f(z)$ and $g(z)$ are analytic in $U$, we say that $f(z)$ is subordinate to $g(z)$, written symbolically $f(z) \prec g(z)$, if there exists a Schwarz function $w(z)(|w(z)| \leq|z|$ in $U)$, such that $f(z)=g(w(z))(z \in U)$.

For analytic functions

$$
f(z)=\sum_{k=0}^{\infty} a_{k} z^{k} \quad \text { and } \quad g(z)=\sum_{k=0}^{\infty} b_{k} z^{k},
$$

by $(f * g)(z)$ we denote the Hadamard product (or convolution) of $f(z)$ and $g(z)$, defined by

$$
(f * g)(z)=\sum_{k=0}^{\infty} a_{k} b_{k} z^{k} .
$$

Let $\alpha_{1}, A_{1}, \ldots, \alpha_{q}, A_{q}$ and $\beta_{1}, B_{1}, \ldots, \beta_{s}, B_{s}(q, s \in N=\{1,2, \ldots\})$ be positive real parameters such that

$$
1+\sum_{k=1}^{s} B_{k}-\sum_{k=1}^{q} A_{k} \geq 0
$$

The Wright generalized hypergeometric function [23] (see also [6], [12] and [19])

$$
\begin{aligned}
& { }_{q} \Psi_{s}\left[\left(\alpha_{1}, A_{1}\right), \ldots,\left(\alpha_{q}, A_{q}\right) ;\left(\beta_{1}, B_{1}\right), \ldots,\left(\beta_{s}, B_{s}\right) ; z\right] \\
& \quad={ }_{q} \Psi_{s}\left[\left(\alpha_{n}, A_{n}\right){ }_{1, q} ;\left(\beta_{n}, B_{n}\right)_{1, s} ; z\right]
\end{aligned}
$$

is defined by

$$
\begin{aligned}
& { }_{q} \Psi_{s}\left[\left(\alpha_{k}, A_{k}\right)_{1, q} ;\left(\beta_{k}, B_{k}\right)_{1, s} ; z\right] \\
& =\sum_{k=0}^{\infty}\left\{\prod_{n=1}^{q} \Gamma\left(\alpha_{n}+k A_{n}\right)\right\}\left\{\prod_{n=1}^{s} \Gamma\left(\beta_{n}+k B_{n}\right)\right\}^{-1} \frac{z^{k}}{k !}(z \in U) .
\end{aligned}
$$

If $A_{n}=1(n=1, \ldots, q)$ and $B_{n}=1(n=1, \ldots, s)$, we have the relationship:

$$
\Omega_{q} \Psi_{s}\left[\left(\alpha_{n}, 1\right)_{1, q} ;\left(\beta_{n}, 1\right)_{1, s} ; z\right]={ }_{q} F_{s}\left(\alpha_{1}, \ldots, \alpha_{q} ; \beta_{1}, \ldots, \beta_{s} ; z\right),
$$

where ${ }_{q} F_{s}\left(\alpha_{1}, \ldots, \alpha_{q} ; \beta_{1}, \ldots, \beta_{s} ; z\right)$ is the generalized hypergeometric function (see for details the books on special functions, as [12], [19]) and

$$
\Omega=\left(\prod_{n=1}^{q} \Gamma\left(\alpha_{n}\right)\right)^{-1}\left(\prod_{n=1}^{s} \Gamma\left(\beta_{n}\right)\right) .
$$

The Wright generalized hypergeometric functions (2) have been recently involved in the geometric function theory, see [1], [2], [3], [14], [15] and [16], as well as: [7], [8], [9] and [13]. It is a special case of Fox's function (see for example [6], [12] and [19]). 
Using the Wright generalized hypergeometric functions Dziok and Raina [2] defined a function ${ }_{q} \phi_{s}\left[\left(\alpha_{n}, A_{n}\right)_{1, q} ;\left(\beta_{n}, B_{n}\right)_{1, s} ; z\right]$ by

$$
{ }_{q} \phi_{s}\left[\left(\alpha_{n}, A_{n}\right)_{1, q} ;\left(\beta_{n}, B_{n}\right)_{1, s} ; z\right]=\Omega z_{q} \Psi_{s}\left[\left(\alpha_{n}, A_{n}\right)_{1, q} ;\left(\beta_{n}, B_{n}\right)_{1, s} ; z\right]
$$

and introduced the following linear operator

$$
\theta\left[\left(\alpha_{n}, A_{n}\right)_{1, q} ;\left(\beta_{n}, B_{n}\right)_{1, s}\right]: \mathcal{A} \rightarrow \mathcal{A},
$$

defined by the convolution

$$
\theta\left[\left(\alpha_{n}, A_{n}\right)_{1, q} ;\left(\beta_{n}, B_{n}\right)_{1, s}\right] f(z)={ }_{q} \phi_{s}\left[\left(\alpha_{n}, A_{n}\right)_{1, q} ;\left(\beta_{n}, B_{n}\right)_{1, s} ; z\right] * f(z) .
$$

We observe that for a function $f(z)$ of the form (1) we have

$$
\theta\left[\left(\alpha_{n}, A_{n}\right)_{1, q} ;\left(\beta_{n}, B_{n}\right)_{1, s}\right] f(z)=z+\sum_{k=2}^{\infty} \Omega \sigma_{k} a_{k} z^{k},
$$

where $\Omega$ is given by (4) and $\sigma_{k}$ is defined by

$$
\sigma_{k}=\frac{\Gamma\left(\alpha_{1}+A_{1}(k-1)\right) \cdots \Gamma\left(\alpha_{q}+A_{q}(k-1)\right.}{\Gamma\left(\beta_{1}+B_{1}(k-1)\right) \cdots \Gamma\left(\beta_{s}+B_{s}(k-1)\right)(k-1) !} .
$$

If, for convenience, we write

$$
\theta\left[\alpha_{1}\right] f(z)=\theta\left[\left(\alpha_{1}, A_{1}\right), \ldots,\left(\alpha_{q}, A_{q}\right) ;\left(\beta_{1}, B_{1}\right), \ldots,\left(\beta_{s}, B_{s}\right)\right] f(z),
$$

then one can easily verify from the definition (5) that

$$
z A_{1}\left(\theta\left[\alpha_{1}\right] f(z)\right)^{\prime}=\alpha_{1} \theta\left[\alpha_{1}+1\right] f(z)-\left(\alpha_{1}-A_{1}\right) \theta\left[\alpha_{1}\right] f(z) .
$$

Using the linear operator $\theta\left[\alpha_{1}\right]$ Aouf and Dziok [1] defined the class $W(q, s ; A, B, \lambda)$ of functions of the form

$$
f(z)=z-\sum_{k=2}^{\infty} a_{k} z^{k}, \quad\left(a_{k} \geq 0\right)
$$

which also satisfy the following condition:

$$
\begin{aligned}
& \frac{1}{(1-\lambda)}\left(\alpha_{1} \frac{\theta\left[\alpha_{1}+1\right] f(z)}{\theta\left[\alpha_{1}\right] f(z)}+A_{1}(1-\lambda)-\alpha_{1}\right) \prec A_{1} \frac{1+A z}{1+B z} \\
&(0 \leq B \leq 1 ;-B \leq A<B ; 0 \leq \lambda<1) .
\end{aligned}
$$

In particular, for $q=s+1$ and $\alpha_{s+1}=A_{s+1}=1$, we write $W(s ; A, B, \lambda)=$ $W(s+1, s ; A, B, \lambda)$. The class $W(q, s ; A, B, 0)=W(q, s ; A, B)$ was studied by Dziok and Raina [2] (see also [3]).

If $A_{n}=1(n=1, \ldots, q)$ and $B_{n}=1(n=1, \ldots, s)$, then we note that $W(q, s ; A, B, 0)=V_{2}^{1}(q, s ; A, B)$. This class was studied by Dziok and Srivastava [4] (see also [5]). Putting moreover $\alpha_{1}=n+1, \alpha_{2}=1$ and $\beta_{1}=1$, we have the class $T_{n}(\lambda, \rho)=W(2,1 ;-\rho, \rho, \lambda)$, which was studied by Patel and Acharya [11].

For the class $W(q, s ; A, B, \lambda)$ we have following result. 
Lemma 1 ([1]). A function $f(z)$ of the form (8) belongs to the class $W(q, s ; A, B, \lambda)$ if and only if

$$
\sum_{k=2}^{\infty} \Omega \delta_{k} a_{k} \leq(B-A)(p-\lambda)
$$

where

$$
\delta_{k}=[(1+B)(k-1)+(B-A)(1-\lambda)] \sigma_{k},
$$

and $\Omega, \sigma_{k}$ are defined by (4) and (6), respectively.

\section{Modified Hadamard PROduct}

For the functions

$$
f_{j}(z)=z-\sum_{k=2}^{\infty} a_{k, j} z^{k} \quad\left(a_{k, j} \geq 0 ; j=1,2\right),
$$

we denote by $\left(f_{1} \otimes f_{2}\right)(z)$ the modified Hadamard product or convolution of the functions $f_{1}$ and $f_{2}$ defined by

$$
\left(f_{1} \otimes f_{2}\right)(z)=z-\sum_{k=2}^{\infty} a_{k, 1} a_{k, 2} z^{k} .
$$

Theorem 1. Let the functions $f_{j}(z)(j=1,2)$ defined by (11) be in the class $W(q, s ; A, B, \lambda)$. If the sequence $\left\{\delta_{k}\right\}$ is nondecreasing, then $\left(f_{1} \otimes\right.$ $\left.f_{2}\right)(z) \in W(q, s ; A, B, \gamma)$, where

$$
\gamma=1-\frac{(1+B)(B-A)(1-\lambda)^{2}}{\Omega[(1+B)+(B-A)(1-\lambda)]^{2} \sigma_{2}-(B-A)^{2}(1-\lambda)^{2}} .
$$

The result is sharp.

Proof. We need to find the largest $\gamma$ such that

$$
\sum_{k=2}^{\infty} \frac{\Omega[(1+B)(k-1)+(B-A)(1-\gamma)] \sigma_{k}}{(B-A)(1-\gamma)} a_{k, 1} a_{k, 2} \leq 1 .
$$

By Lemma 1 and the Cauchy-Schwarz inequality, we obtain

$$
\sum_{k=2}^{\infty} \frac{\Omega[(1+B)(k-1)+(B-A)(1-\lambda)] \sigma_{k}}{(B-A)(1-\lambda)} \sqrt{a_{k, 1} a_{k, 2}} \leq 1 .
$$

Thus by (13) it is sufficient to show that

$$
\sqrt{a_{k, 1} a_{k, 2}} \leq \frac{[(1+B)(k-1)+(B-A)(1-\lambda)](1-\gamma)}{[(1+B)(k-1)+(B-A)(1-\gamma)](1-\lambda)} \quad(k \geq 2) .
$$


By (14) we have

$$
\sqrt{a_{k, 1} a_{k, 2}} \leq \frac{(B-A)(1-\lambda)}{\Omega[(1+B)(k-1)+(B-A)(1-\lambda)] \sigma_{k}} \quad(k \geq 2) .
$$

Consequently, we need only to prove that

$$
\begin{array}{r}
\gamma \leq 1-\frac{(k-1)(1+B)(B-A)(1-\lambda)^{2}}{\Omega[(1+B)(k-1)+(B-A)(1-\lambda)]^{2} \sigma_{k}-(B-A)^{2}(1-\lambda)^{2}} \\
\quad(k \geq 2) .
\end{array}
$$

Since

$$
\begin{aligned}
& \Phi(k) \\
& =1-\frac{(k-1)(1+B)(B-A)(1-\lambda)^{2}}{\Omega[(1+B)(k-1)+(B-A)(1-\lambda)]^{2} \sigma_{k}-(B-A)^{2}(1-\lambda)^{2}}
\end{aligned}
$$

is an increasing function of $k(k \geq 2)$, letting $k=2$ in (15), we obtain

$$
\gamma \leq \Phi(2)=1-\frac{(1+B)(B-A)(1-\lambda)^{2}}{\Omega[(1+B)+(B-A)(1-\lambda)]^{2} \sigma_{2}-(B-A)^{2}(1-\lambda)^{2}},
$$

which proves the main assertion of Theorem 1. Finally, by taking the functions $f_{j}(z)(j=1,2)$ given by

$$
f_{j}(z)=z-\frac{(B-A)(1-\lambda)}{\Omega[(1+B)+(B-A)(1-\lambda)] \sigma_{2}} z^{2} \quad(j=1,2),
$$

we can see that the result is sharp.

Theorem 2. Let the functions $f_{j}(z)(j=1,2)$ defined by (11) be in the class $W(q, s ; A, B, \lambda)$. If the sequence $\left\{\delta_{k}\right\}$ is nondecreasing, then the function

$$
h(z)=z-\sum_{k=2}^{\infty}\left(a_{k, 1}^{2}+a_{k, 2}^{2}\right) z^{k}
$$

belongs to the class $W(q, s ; A, B, \tau)$, where

$$
\tau=1-\frac{2(1+B)(B-A)(1-\lambda)^{2}}{\Omega[(1+B)+(B-A)(1-\lambda)]^{2} \sigma_{2}-2(B-A)^{2}(1-\lambda)^{2}} .
$$

The result is sharp for the functions $f_{j}(z)(j=1,2)$ defined by (16).

Proof. By Lemma 1, we obtain

$$
\sum_{k=2}^{\infty}\left\{\frac{\Omega[(1+B)(k-1)+(B-A)(1-\lambda)] \sigma_{k}}{(B-A)(1-\lambda)}\right\}^{2} a_{k, 1}^{2}
$$




$$
\leq\left\{\sum_{k=2}^{\infty} \frac{\Omega[(1+B)(k-1)+(B-A)(1-\lambda)] \sigma_{k}}{(B-A)(1-\lambda)} a_{k, 1}\right\}^{2} \leq 1
$$

and

$$
\begin{aligned}
& \sum_{k=2}^{\infty}\left\{\frac{\Omega[(1+B)(k-1)+(B-A)(1-\lambda)] \sigma_{k}}{(B-A)(1-\lambda)}\right\}^{2} a_{k, 2}^{2} \\
& \leq\left\{\sum_{k=2}^{\infty} \frac{\Omega[(1+B)(k-1)+(B-A)(1-\lambda)] \sigma_{k}}{(B-A)(1-\lambda)} a_{k, 2}\right\}^{2} \leq 1 .
\end{aligned}
$$

It follows from (18) and (19) that

$$
\sum_{k=2}^{\infty} \frac{1}{2}\left\{\frac{\Omega[(1+B)(k-1)+(B-A)(1-\lambda)] \sigma_{k}}{(B-A)(1-\lambda)}\right\}^{2}\left(a_{k, 1}^{2}+a_{k, 2}^{2}\right) \leq 1 .
$$

Therefore, we need to find the largest $\tau$ such that

$$
\begin{aligned}
& \frac{\Omega[(1+B)(k-1)+(B-A)(1-\tau)] \sigma_{k}}{(B-A)(1-\tau)} \\
& \leq \frac{1}{2}\left\{\frac{\Omega[(1+B)(k-1)+(B-A)(1-\lambda)] \sigma_{k}}{(B-A)(1-\lambda)}\right\}^{2} \quad(k \geq 2),
\end{aligned}
$$

that is,

$$
\tau \leq 1-\frac{2(k-1)(1+B)(B-A)(1-\lambda)^{2}}{\Omega[(1+B)(k-1)+(B-A)(1-\lambda)]^{2} \sigma_{k}-2(B-A)^{2}(1-\lambda)^{2}}
$$

Since

$$
D(k)=1-\frac{2(k-1)(1+B)(B-A)(1-\lambda)^{2}}{\Omega[(1+B)(k-1)+(B-A)(1-\lambda)]^{2} \sigma_{k}-2(B-A)^{2}(1-\lambda)^{2}},
$$

is an increasing function of $k(k \geq 2)$, we readily have

$$
\tau \leq D(2)=1-\frac{2(1+B)(B-A)(1-\lambda)^{2}}{\Omega[(1+B)+(B-A)(1-\lambda)]^{2} \sigma_{2}-2(B-A)^{2}(1-\lambda)^{2}},
$$

and Theorem 2 follows at once.

Putting $\lambda=0$ in Theorems 1 and 2, we obtain the following two corollaries.

Corollary 1. Let the functions $f_{j}(z)(j=1,2)$ defined by (11) be in the class $W(q, s ; A, B)$. If the sequence $\left\{\delta_{k}\right\}$ is nondecreasing, then $\left(f_{1} \otimes f_{2}\right)(z) \in$ $W(q, s ; A, B, \gamma)$, where

$$
\gamma=1-\frac{(1+B)(B-A)}{\Omega(1+2 B-A)^{2} \sigma_{2}-(B-A)^{2}} .
$$


The result is sharp.

Corollary 2. Let the functions $f_{j}(z)(j=1,2)$ defined by (11) be in the class $W(q, s ; A, B)$. If the sequence $\left\{\delta_{k}\right\}$ is nondecreasing, then the function $h(z)$ defined by (17) belongs to the class $W(q, s ; A, B, \tau)$, where

$$
\tau=1-\frac{2(1+B)(B-A)}{\Omega(1+2 B-A)^{2} \sigma_{2}-2(B-A)^{2}} .
$$

The result is sharp.

Taking $q=2, s=1, A_{1}=A_{2}=B_{1}=1, \alpha_{1}=n+1 \in N, \alpha_{2}=\beta_{1}=1$, $B=-A=\rho(0<\rho \leq 1)$ in Theorems 1 and 2 , respectively, we obtain the following consequences:

Corollary 3. Let the functions $f_{j}(z)(j=1,2)$ defined by (11) be in the class $T_{n}(\lambda, \rho)$. Then $\left(f_{1} \otimes f_{2}\right)(z) \in T_{n}(\gamma, \rho)$, where

$$
\gamma=1-\frac{2 \rho(1+\rho)(1-\lambda)^{2}}{[1+\rho(3-2 \lambda)]^{2}(n+1)-4 \rho^{2}(1-\lambda)^{2}} .
$$

The result is sharp.

Corollary 4. Let the functions $f_{j}(z)(j=1,2)$ defined by (11) be in the class $T_{n}(\lambda, \rho)$. Then the function $h(z)$ defined by $(17)$ belongs to the class $T_{n}(\tau, \rho)$, where

$$
\tau=1-\frac{4 \rho(1+\rho)(1-\lambda)^{2}}{[1+\rho(3-2 \lambda)]^{2}(n+1)-8 \rho^{2}(1-\lambda)^{2}} .
$$

The result is sharp.

\section{Definitions AND APPLiCATIONS OF FRACTIONAL CALCUlus}

We start with some definitions of fractional calculus operators (that is fractional derivatives and fractional integrals), as adopted for use in classes of analytic functions. First we recall the definition of Saigo operator [17], [18] (see also [21]).

For real numbers $\gamma, \zeta$ and $\eta$, the fractional derivative-integral operator $I_{0, z}^{\gamma, \zeta, \eta}$ is defined by

$$
\begin{aligned}
& I_{0, z}^{\gamma, \zeta, \eta} f(z)=\frac{z^{-\gamma-\zeta}}{\Gamma(\gamma)} \int_{0}^{z}(z-t)^{\gamma-1}{ }_{2} F_{1}\left(\gamma+\zeta,-\eta ; \gamma ; 1-\frac{t}{z}\right) f(t) d t \quad \text { for } \gamma>0 \\
& I_{0, z}^{\gamma, \zeta, \eta} f(z)=\frac{d^{n}}{d z^{n}} I_{0, z}^{\gamma+n, \zeta-\eta, \eta-n} f(z) \quad \text { for } 0<\gamma+n \leq 1, n=1,2, \ldots
\end{aligned}
$$


where $f(z)$ is an analytic function in a simply connected region of the $z$-plane containing the origin with the order

$$
f(z)=O\left(|z|^{\varepsilon}\right) \quad(z \rightarrow 0)
$$

where $\varepsilon>\max (0, \zeta-\eta)-1$ and the multiplicity of $(z-t)^{\gamma-1}$ is removed by requiring $\log (z-t)$ to be real when $z-t>0$. The function ${ }_{2} F_{1}(a, b ; c ; z)$ is the Gaussian hypergeometric function (3) defined, in terms of the Pochhammer symbol

$$
(\lambda)_{n}= \begin{cases}1 & (n=0) \\ \lambda(\lambda+1) \cdots(\lambda+n-1) & (n \in \mathbb{N}),\end{cases}
$$

by

$$
{ }_{2} F_{1}(a, b ; c ; z)=\sum_{n=0}^{\infty} \frac{(a)_{n}(b)_{n}}{(c)_{n}} \frac{z^{n}}{n !} \quad(z \in U) .
$$

In particular we obtain the fractional derivative-integral operator

$$
D_{z}^{\gamma} f(z)=I_{0, z}^{\gamma, \gamma, \eta} f(z)
$$

introduced by Owa [10] (see also Srivastava and Owa [20]).

In order to prove our result for the operator $I_{0, z}^{\gamma, \zeta, \eta}$, we recall here the following lemma.

Lemma $2([22])$. If $n>\max \{0, \zeta-\eta\}-1$, then

$$
I_{0, z}^{\gamma, \zeta, \eta} z^{n}=\frac{\Gamma(n+1) \Gamma(n-\zeta+\eta+1)}{\Gamma(n-\zeta+1) \Gamma(n+\gamma+\eta+1)} z^{n-\zeta}
$$

We can prove easy the following theorem.

Theorem 3. Let $l \in N \cup\{0\},-\gamma \leq \zeta \leq l, \zeta<2+\eta$, and let the sequence $\left\{\delta_{k} /(k)_{l}\right\}$ be nondecreasing. If the function $f(z)$ is in the class $W(q, s ; A, B, \lambda)$ and $0<|z|<1$, then

$$
\begin{aligned}
& \left|I_{0, z}^{\gamma, \zeta, \eta} f(z)\right| \geq \frac{\Gamma(2-\zeta+\eta)|z|^{1-\zeta}}{\Gamma(2-\zeta) \Gamma(2+\gamma+\eta)}\left\{1-\frac{2(B-A)(1-\lambda)(2-\zeta+\eta)}{\Omega \delta_{2}(2-\zeta)(2+\gamma+\eta)}|z|\right\}, \\
& \left|I_{0, z}^{\gamma, \zeta, \eta} f(z)\right| \leq \frac{\Gamma(2-\zeta+\eta)|z|^{1-\zeta}}{\Gamma(2-\zeta) \Gamma(2+\gamma+\eta)}\left\{1+\frac{2(B-A)(1-\lambda)(2-\zeta+\eta)}{\Omega \delta_{2}(2-\zeta)(2+\gamma+\eta)}|z|\right\} .
\end{aligned}
$$

The result is sharp for extremal function $f$ of the form

$$
f(z)=z-\frac{(B-A)(1-\lambda)}{\Omega \delta_{2}} z^{2}
$$

Putting $\zeta=\gamma$ in Theorem 3 and using (20) we obtain the following corollary. 
Corollary 5. Let $l \in N \cup\{0\}, \gamma \geq-l$ and let the sequence $\left\{\delta_{k} /(k)_{l}\right\}$ be nondecreasing. If the function $f(z)$ is in the class $W(q, s ; A, B, \lambda)$ and $0<|z|<1$, then

$$
\begin{aligned}
& \left|D_{z}^{\gamma} f(z)\right| \geq \frac{|z|^{1-\gamma}}{\Gamma(2-\gamma)}\left\{1-\frac{2(B-A)(1-\lambda)}{\Omega \delta_{2}(2-\gamma)}|z|\right\}, \\
& \left|D_{z}^{\gamma} f(z)\right| \leq \frac{|z|^{1-\gamma}}{\Gamma(2-\gamma)}\left\{1+\frac{2(B-A)(1-\lambda)}{\Omega \delta_{2}(2-\gamma)}|z|\right\} .
\end{aligned}
$$

The result is sharp for extremal function $f$ of the form (21).

Corollary 6. Let $l \in N \cup\{0\}, \gamma \geq-l$ and let the sequence $\left\{\delta_{k} /(k)_{l}\right\}$ be nondecreasing. If the function $f(z)$ is in the class $W(q, s ; A, B, \lambda)$, then $D_{z}^{\gamma} f(z)$ is included in a disc with its center at the origin and radius $r$ given by

$$
r=\frac{1}{\Gamma(2-\gamma)}\left\{1+\frac{2(B-A)(1-\lambda)}{\Omega \delta_{2}(2-\gamma)}\right\}
$$

Remark 1. Taking $\lambda=0$ in Theorem 3 and Corollaries 5 and 6 , respectively, we obtain the results for the class $W(q, s ; A, B, 0)=W(q, s ; A, B)$ studied by Dziok and Raina [2]. Corollaries 1 and 2 are improvements of the results obtained by Patel and Acharya [11].

Acknowledgements. The authors express their sincerest thanks to the referees for useful comments.

\section{References}

[1] Aouf, M. K., Dziok, J., Certain class of analytic functions associated with the Wright generalized hypergeometric function, J. Math. Appl. 30 (2008), 23-32.

[2] Dziok, J., Raina, R. K., Families of analytic functions associated with the Wright generalized hypergeometric function, Demonstratio Math. 37(3) (2004), 533-542.

[3] Dziok, J., Raina, R. K., Srivastava, H. M., Some classes of analytic functions associated with operators on Hilbert space involving Wright's generalized hypergeometric function, Proc. Jangieon Math. Soc. 7 (2004), 43-55.

[4] Dziok, J., Srivastava, H. M., Certain subclasses of analytic functions associated with the generalized hypergeometric function, Integral Transforms Spec. Funct. 14 (2003), 7-18.

[5] Dziok, J., Srivastava, H. M., Classes of analytic functions associated with the generalized hypergeometric function, Appl. Math. Comput. 103 (1999), 1-13.

[6] Kiryakova, V., Generalized Fractional Calculus and Applications, Longman \& J. Wiley, Harlow-New York, 1994.

[7] Kiryakova, V., Saigo, M., Criteria for generalized fractional integrals to preserve univalency of analytic functions, C. R. Acad. Bulgare Sci. 58(10) (2005), 1127-1134. 
[8] Kiryakova, V., Saigo, M., Owa, S., Distortion and characterization theorems for generalized fractional integration operators involving $H$-function in subclasses of univalent functions, Fukuoka Univ. Sci. Rep. 34(1) (2004), 1-16.

[9] Kiryakova, V., Saigo, M., Srivastava, H. M., Some criteria for univalence of analytic functions involving generalized fractional calculus operators, Fract. Calc. Appl. Anal. 1(1) (1998), 79-104.

[10] Owa, S., On the distortion theorems. I, Kyungpook Math. J. 18 (1978), 53-59.

[11] Patel, J., Acharya, M., Certain subclasses of starlike functions with negative coefficients, Bull. Calcuta Math. Soc. 87 (1995), 265-276.

[12] Prudnikov, A. P., Brychkov, Yu. A., Marichev, O. I., Integrals and Series. Vol. 3: More Special Functions, Gordon \& Breach Sci. Publ., New York, 1990.

[13] Raina, R. K., Kalia, R. N., Characterizations for subclasses of analytic functions connecting linear fractional calculus operators, Fract. Calc. Appl. Anal. 1(4) (1998), 335-350.

[14] Raina, R. K., Nahar, T. S., A note on boundedness properties of Wright's generalized hypergeometric function, Ann. Math. Blaise Pascal 4 (1997), 83-95.

[15] Raina, R. K., Nahar, T. S., On characterization of certain Wright's generalized hypergeometric functions involving certain subclasses of analytic functions, Informatica (Vilnius) 10 (1999), 219-230.

[16] Raina, R. K., Nahar, T. S., On univalent and starlike Wright's hypergeometric functions, Rend. Sem. Mat. Univ. Padova 95 (1996), 11-22.

[17] Saigo, M., A certain boundary value problem for the Euler-Darboux equation, Math. Japon. 24 (1980), 377-385.

[18] Saigo, M., A remark on integral operators involving the Gauss hypergeometric functions, Math. Rep. Kyushu Univ. 11 (1978), 135-143.

[19] Srivastava, H. M., Gupta, C. K., Goyal, S. P., The H-Functions of One and Two Variables with Applications, South Asian Publ., New Delhi, 1982.

[20] Srivastava, H. M., Owa, S., An application of the fractional derivative, Math. Japon. 29 (1984), 383-389.

[21] Srivastava, H. M., Owa, S., (editors), Current Topics in Analytic Function Theory, World Sci. Publ. Comp., Singapore-New Jersey-London-Hong Kong, 1992.

[22] Srivastava, H. M., Saigo, M., Owa, S., A class of distortion theorems involving certain operators of fractional calculus, J. Math. Anal. Appl. 131 (1988), 412-420.

[23] Wright, E. M., The asymptotic expansion of the generalized hypergeometric function, Proc. London Math. Soc. (3) 46 (1946), 389-408.

Mohamed Kamal Aouf

Department of Mathematics

Faculty of ScIEnce

MANSOURA UnIVERSITY

MANSOURA 35516, EgYPT

E-MAIL: MKAOUF127@YAHOO.COM
JACEK DZIOK

InSTITUTE OF MATHEMATICS UNIVERSITY OF RZESZOW

Rejtana 16A

PL-35-310 Rzeszow, Poland

E-MAIL: JDZIOK@UNIV.RZESZOW.PL 